\title{
Eigenstructures of MIMO Fading Channel Correlation Matrices and Optimum Linear Precoding Designs for Maximum Ergodic Capacity
}

\author{
Hamid Reza Bahrami and Tho Le-Ngoc \\ Department of Electrical and Computer Engineering, McGill University, 3480 University Street, Montréal, QC, Canada H3A 2A7
}

Received 27 October 2006; Revised 10 February 2007; Accepted 25 March 2007

Recommended by Nicola Mastronardi

The ergodic capacity of MIMO frequency-flat and -selective channels depends greatly on the eigenvalue distribution of spatial correlation matrices. Knowing the eigenstructure of correlation matrices at the transmitter is very important to enhance the capacity of the system. This fact becomes of great importance in MIMO wireless systems where because of the fast changing nature of the underlying channel, full channel knowledge is difficult to obtain at the transmitter. In this paper, we first investigate the effect of eigenvalues distribution of spatial correlation matrices on the capacity of frequency-flat and -selective channels. Next, we introduce a practical scheme known as linear precoding that can enhance the ergodic capacity of the channel by changing the eigenstructure of the channel by applying a linear transformation. We derive the structures of precoders using eigenvalue decomposition and linear algebra techniques in both cases and show their similarities from an algebraic point of view. Simulations show the ability of this technique to change the eigenstructure of the channel, and hence enhance the ergodic capacity considerably.

Copyright (c) 2007 H. R. Bahrami and T. Le-Ngoc. This is an open access article distributed under the Creative Commons Attribution License, which permits unrestricted use, distribution, and reproduction in any medium, provided the original work is properly cited.

\section{INTRODUCTION}

It has been shown that the capacity of a MIMO system is greatly reduced by spatial correlation in the underlying channel $[1,2]$. Spatial correlation can reduce the rank of the channel matrix, and hence greatly surpasses the multiplexing gain of a MIMO system. Various techniques that have been proposed in the literature to reduce the correlation effects are based on two main approaches. One aims to avoid correlation in the channel by antenna beamforming $[3,4]$. The other tries to cancel the existing channel correlation by suitable methods at the transmitter or receiver. In this paper, our focus is on the linear precoding technique based on the knowledge of correlation at the transmitter, aiming to increase the ergodic capacity of fading channels by modifying the eigenvalue spread of the channel correlation matrices.

Linear precoder design in MIMO systems is a relatively simple (in term of implementation and design complexity) strategy that tries to improve the transmission quality and rate by optimal allocation of resources such as power and bits over multiple antennas, based on the channel properties. Design of the precoders based on full channel knowledge for MIMO systems in frequency-flat and -selective channels has been investigated by many works. For a detailed overview on the designs for frequency-flat channels, see $[5,6]$. While we see a number of different precoder structures for frequencyflat fading channel proposed in the literature, there are fewer papers addressing MIMO precoding designs in a frequencyselective fading environment [7]. In designs based on full channel knowledge, it is assumed that the transmitter has the instantaneous channel information and based on this information, a metric related to performance, such as pairwise error probability (PEP) or minimum mean-square error (MMSE) or rate (ergodic capacity or probability of outage), is defined and optimized by selection of proper linear precoder.

In a fast fading environment, however, the assumption of full channel knowledge at the transmitter is no longer realistic due to the finite delay in channel response estimation and reporting. Hence, it is more reasonable to assume that the transmitter knows only partial channel knowledge such as spatial correlation information, that is, transmit and receive correlation matrices.

Optimal precoding designs using PEP criterion based on transmit and receive correlation matrices were presented in $[8,9]$, respectively. In [10], optimal precoding designs based 
on both transmit and receive correlation matrices were developed for three different criteria, that is, PEP, MMSE, and ergodic capacity. The results indicate that the optimal precoder structures for these criteria are very similar. All of the above designs are for flat fading channels.

In this paper, we investigate the channel correlation effects on the capacity of frequency-flat and frequencyselective fading channels from an algebraic viewpoint and develop the corresponding linear precoding structures to maximize their ergodic capacity. We show that the eigenvalues of the correlation matrices play a key role in the ergodic capacity of fading channels. In particular, the effect of correlation on the capacity of the system becomes more pronounced with increase in the eigenvalue spread of the spatial correlation matrices. Therefore, in general, our focus is to find how we can modify the eigenvalues of the channel correlation matrices to enhance the capacity. Based on linear algebraic structures of frequency-flat and frequencyselective fading MIMO channels, we construct suitable analytical models to include channel spatial correlations in both cases, and derive the precoding matrix structures that can maximize their ergodic channel capacity. In both cases, the precoding matrices are closely related to the eigenstructures (eigenvalues and eigenvectors) of spatial correlation matrices. We further show that the structure of the precoder in the frequency-flat case is an eigenbeamformer with beams pointing to the eigenmodes of the transmit correlation matrix. For a frequency-selective fading channel with $L$ independent effective paths, the precoder can be constructed as a number of parallel precoders for frequency-flat fading channels. In this sense, there is a kind of duality between precoder design for frequency-flat and -selective channels.

The rest of this paper is organized as follows. In Section 2, we consider the case of frequency-flat fading channels. The so-called Kronecker model is introduced to represent the spatial correlation effects in MIMO system. Based on this channel model, we investigate the effects of eigenvalues of spatial correlation matrix and their spread on the ergodic channel capacity and develop the corresponding precoding structure based on the eigenstructure of the channel spatial correlation matrix in order to maximize the ergodic capacity. In Section 3, we consider the case of frequency-selective fading channels. We develop a comprehensive linear algebraic model of a frequency-selective fading channel with $L$ effective paths in terms of channel correlation matrices. Furthermore, we analyze the effects of the eigenstructures of the channel correlation matrices on the ergodic capacity of a frequencyselective fading channel and develop the optimum precoder based on the eigenstructures of spatial correlation matrices of $L$ effective channel paths for maximum ergodic capacity. It is shown that the structure includes $L$ parallel precoders, each for one frequency-flat fading channel path, with specific power loadings. Section 4 presents illustrative examples with numerical results and plots. The effects of eigenvalues of spatial correlation matrices on the capacity of both frequencyflat and frequency-selective fading channels in various conditions are presented. The effects of precoding on the eigenvalue spreads of the channel correlation matrices are also shown. Furthermore, performance in terms of achievable ergodic capacity versus SNR of the proposed precoders is evaluated and compared with that of systems using no precoding in various scenarios by means of simulation. It is shown that the precoders perform well in changing the eigenstructure (mainly eigenvalue spread) of the channels in favor of channel capacity. In other words, the precoders are capable of providing a considerable capacity gain in different propagation scenarios by changing the characteristics of the channel correlation matrices. Finally, Section 5 includes with concluding remarks.

\section{FREQUENCY-FLAT FADING CHANNEL}

\subsection{System model}

Consider a MIMO transmission system over a frequency-flat fading channel, using transmitter and receiver equipped with $M$ and $N$ antennas, respectively. The discrete-time wireless MIMO fading channel impulse response can be assumed to be an $N \times M$ matrix $\mathbf{H}$ and the system model (input-output relationship) at the $k$ th time instant can be written as

$$
\mathbf{y}(k)=\mathbf{H}(k) \cdot \mathbf{s}(k)+\mathbf{n}(k),
$$

where $\mathbf{s}[k]$ is the transmitted $M \times 1$ data vector with statistically independent entries and $\mathbf{y}[k]$ and $\mathbf{n}[k]$ denote the $N \times 1$ received and noise vectors, respectively. We assume that the elements of $\mathbf{H}$ and $\mathbf{n}$ are complex Gaussian random variables with $1 / 2$ variance per dimension, and $E\left\{\mathbf{n} \mathbf{n}^{H}\right\}=\sigma_{n}^{2} \mathbf{I}_{N}$, where $\sigma_{n}^{2}$ is the noise variance and $\mathbf{I}_{N}$ is the identity matrix of size $N$. Besides, $E\{\cdot\}$ denotes expectation and superscript $H$ is Hermitian (conjugate transpose) operator.

For simplicity, we assume the receiver (e.g., mobile unit) to be surrounded by local scatterers so that fading at the mobile unit is spatially uncorrelated while transmitter (e.g., base station) is located in a high altitude, and therefore the fading is correlated at base station. However, it is straightforward to generalize the model to the case when both transmitter and receiver are spatially correlated. ${ }^{1}$ Due to the assumed spatial correlation at transmit side, the elements of each row of $\mathbf{H}$ are correlated and for each row, we can write

$$
\mathbf{R}_{T}=E\left\{\mathbf{h}_{i}^{H} \mathbf{h}_{i}\right\}, \quad i=0 \cdots N-1,
$$

where $\mathbf{h}_{i}$ is the $i$ th row of $\mathbf{H}$. $\mathbf{R}_{T}$ is the $M \times M$ transmit nonnegative, semidefinite correlation matrix, and hence can be represented as $\mathbf{R}_{T}=\mathbf{R}_{T}^{1 / 2} \mathbf{R}_{T}^{H / 2}$ (Choleski factorization). Subsequently, the channel matrix $\mathbf{H}$ can be represented as

$$
\mathbf{H}=\mathbf{G R}_{T}^{1 / 2},
$$

where $\mathbf{G}$ is an uncorrelated $N \times M$ matrix with i.i.d. zeromean normalized Gaussian distributed entries, that is, $\mathbf{G} \sim$ $C N(0,1)$. The model proposed here is usually known as Kronecker model in the related literature [11].

\footnotetext{
${ }^{1}$ For a more detailed analysis including receive correlation, see [10].
} 


\subsection{Effect of correlation}

We start by defining the mutual information in each channel use. We assume an independent and invariant realization of the channel matrix in each channel. Using (1), the mutual information of such a system is defined as $[12,13]$

$$
I(\mathbf{s} ; \mathbf{y})=\log \left[\operatorname{det}\left(\mathbf{I}_{N}+\frac{1}{\sigma_{n}^{2}} \mathbf{H} \mathbf{\Sigma} \mathbf{H}^{H}\right)\right],
$$

where $\Sigma$ is the $M \times M$ covariance matrix of Gaussian input $\mathbf{x}$ with a maximum power limit due to the total power limitation at transmitter, that is, $\operatorname{tr}(\boldsymbol{\Sigma}) \leq P$. Note that the instantaneous capacity of the system is defined as the maximum of mutual information over all covariance matrices $\Sigma$ that satisfy the power constraint, and the ergodic capacity is the ensemble average over instantaneous capacity.

In the following analysis, for simplicity we assume an equal power allocation $\boldsymbol{\Sigma}=(P / N) \mathbf{I}_{M}$. Based on this assumption, the mutual information in (4) can be written as

$$
I(\mathbf{s} ; \mathbf{y})=\log \left[\operatorname{det}\left(\mathbf{I}_{N}+\frac{P}{N \sigma_{n}^{2}} \mathbf{H} \mathbf{H}^{H}\right)\right] .
$$

This is in fact the instantaneous channel capacity when transmitter has no knowledge about the channel. Our objective is to understand the effect of transmit correlation matrix (more specifically its eigenvalues) on the ergodic capacity of the system.

Lemma 1. Instantaneous mutual information has the following distribution:

$$
I \sim \log \operatorname{det}\left(\mathbf{I}_{M}+\frac{P}{N \sigma_{n}^{2}} \Delta \mathbf{G}^{H} \mathbf{G}\right),
$$

where $\Delta=\operatorname{diag}\left\{\delta_{i}\left(\mathbf{R}_{T}\right)\right\}_{i=0}^{M-1}$, that is, $\delta_{i}$ 's are the eigenvalues of transmit correlation matrix.

Proof. Substituting $\mathbf{H}$ from (3) into (5) will result in

$$
I=\log \left[\operatorname{det}\left(\mathbf{I}_{N}+\frac{P}{N \sigma_{n}^{2}} \mathbf{G R}_{T} \mathbf{G}^{H}\right)\right] .
$$

By applying the eigenvalue decomposition of $\mathbf{R}_{T}=\boldsymbol{\Phi} \Delta \boldsymbol{\Phi}^{H}$ and the fact that $\mathbf{G} \boldsymbol{\Phi} \sim \mathbf{G}$ (and hence $\boldsymbol{\Phi}^{H} \mathbf{G}^{H} \sim \mathbf{G}^{H}$ ), (7) can be rewritten as

$$
I \sim \log \operatorname{det}\left(\mathbf{I}_{N}+\frac{P}{N \sigma_{n}^{2}} \mathbf{G} \Delta \mathbf{G}^{H}\right) .
$$

Using the matrix equality $\operatorname{det}(\mathbf{I}+\mathbf{A B})=\operatorname{det}(\mathbf{I}+\mathbf{B A})$ will complete the proof.

As previously mentioned, the ergodic capacity is defined as $C=E\{I\}$. The importance of $C$ comes from the fact that at transmission rates lower than $C$, the error probability of a good code decays exponentially with the transmission rate. Here, our objective is to investigate the effects of the eigenvalues of transmit correlation matrix on $C$. We show the importance of these eigenvalues in two ways. First, following the same approach as in [14], we consider the asymptotic case of large number of receive antennas, based on the law of large numbers, when the number of receive antennas $(N)$ is large $(1 / N) \mathbf{G}^{H} \mathbf{G} \rightarrow \mathbf{I}_{M}$, and is hence in the limit

$$
\begin{aligned}
C & =E\left\{\log \operatorname{det}\left(\mathbf{I}_{M}+\frac{P}{N \sigma_{n}^{2}} \Delta \mathbf{G}^{H} \mathbf{G}\right)\right\} \\
& =\log \operatorname{det}\left(\mathbf{I}_{M}+\frac{P}{\sigma_{n}^{2}} \Delta\right) \\
& =\sum_{i=1}^{M} \log \left(1+\frac{P}{\sigma_{n}^{2}} \delta_{i}\right),
\end{aligned}
$$

where $\delta_{i}$ 's are the diagonal entries of $\Delta$, the eigenvalue matrix check of transmit correlation matrix $\mathbf{R}_{T}$. This clearly shows the effect of the eigenvalues of correlation matrix on the ergodic capacity of a frequency-flat MIMO channel. It is also possible to derive the same result by applying Jensen's inequality [15] to the first equation in (9) to compute an upper bound on ergodic capacity. Using Jensen's inequality,

$$
C \leq C_{\mathrm{UB}}=\log \operatorname{det} E\left\{\left(\mathbf{I}_{M}+\frac{P}{N \sigma_{n}^{2}} \Delta \mathbf{G}^{H} \mathbf{G}\right)\right\} .
$$

Since the entries of $\mathbf{G}$ are Gaussian with zero mean and 1/2 variance per dimension, it follows that

$$
\begin{aligned}
C \leq C_{\mathrm{UB}} & =\log \operatorname{det} E\left\{\left(\mathbf{I}_{M}+\frac{P}{\sigma_{n}^{2}} \Delta\right)\right\} \\
& =\sum_{i=1}^{M} \log \left(1+\frac{P}{\sigma_{n}^{2}} \delta_{i}\right) .
\end{aligned}
$$

This bound gets tighter by increasing $N$, the number of the receive antennas, and the inequality in (11) becomes equality in the limit of large $N$.

The following lemma specifies the optimal case for eigenvalues in order to maximize the ergodic capacity. We borrow this lemma from [14].

Lemma 2. For $\operatorname{tr}\left(\mathbf{R}_{T}\right)=1$, the ergodic capacity is maximized when $\delta_{i}=1 / N, i=1 \cdots N$.

The proof is straightforward and can be obtained by symmetry argument.

Lemma 2 shows that the best case is when the channel is indeed uncorrelated, that is, $\mathbf{R}_{T}=(1 / N) \mathbf{I}_{N}$. Now the question is what transmit strategy can be used when the channel is correlated. Our focus here is to devise a linear transmission strategy to maximize the ergodic capacity when the transmit correlation matrix is not identity.

In wireless communications, this question is also appealing from another point of view. Here, we assume that we just know the transmit correlation matrix $\mathbf{R}_{T}$ at the transmitter and do not have information of the channel matrix $\mathbf{H}$. This assumption becomes more important in wireless channels where the channel changes very fast (i.e., fast fading channels), since it is difficult, or sometimes impossible to acquire instantaneous channel response, $\mathbf{H}$, at the transmitter. On the other hand, transmit correlation matrix (or any other 
second-order statistics) of the channel changes much slowly compared to instantaneous channel response, $\mathbf{H}$. Therefore, it is possible in fast fading environment to obtain an accurate transmit correlation matrix at the transmitter. Sometimes in the related literature, such information is called partial channel information. In state-of-the-art communications systems, these types of channel information become more and more important as we are interested in transmitting information to high-speed mobile units.

Our objective through this paper is to apply an $M \times M$ linear transformation (precoding) $\mathbf{W}$ over information symbols $\mathbf{s}$ to get an $M \times 1$ transmit vector $\mathbf{x}$, that is, $\mathbf{x}=\mathbf{W s}$, under the power constraint. The precoding matrix is selected such that a performance metric (e.g., the ergodic capacity) is optimized. We assume that the transmitter is just informed of the transmit correlation matrix $\mathbf{R}_{T}$. We treat the flat-fading channel in this section and the frequency-selective fading cases in the next section.

\subsection{Precoder design}

We assume that the receiver has the perfect channel information but the transmitter knows only spatial and path correlation matrices. Our objective is to design the precoding matrix $\mathbf{W}$ to maximize the ergodic capacity for a given total transmit power. Applying precoding matrix, the ergodic capacity of the MIMO system in a frequency-flat fading channel can now be written as

$$
C=E\left[\log _{2}\left(\operatorname{det}\left(\mathbf{I}_{M}+\frac{1}{N \sigma_{n}^{2}} \mathbf{W}^{H} \mathbf{H}^{H} \mathbf{H} \mathbf{W}\right)\right)\right] .
$$

Note that the power constant $P$ in (7) is now considered in the elements of precoder matrix, and hence a power constraint is applied to its entries, that is, $\operatorname{tr}\left\{\mathbf{W} \mathbf{W}^{H}\right\} \leq P$. Getting expectation from the log-function in (12) is very hard (if not impossible). By applying Jensen's inequality [15] to logdet function, that is, $E\{\log [\operatorname{det}(\mathbf{A})]\} \leq \log [\operatorname{det}(E\{\mathbf{A}\})]$, we can derive an upper bound on the ergodic capacity as

$$
C \leq C_{\mathrm{UB}}=\log _{2}\left(\operatorname{det} E\left(\mathbf{I}_{M}+\frac{1}{N \sigma_{n}^{2}} \mathbf{W}^{H} \mathbf{R}_{T}^{H / 2} \mathbf{G}^{H} \mathbf{G R}_{T}^{1 / 2} \mathbf{W}\right)\right),
$$

where $\mathbf{H}$ has been substituted from (3).

Lemma 3. The optimum precoding matrix for frequency-flat fading channel is directly related to the eigenvector matrix of transmit correlation matrix $\mathbf{R}_{T}$ and can be written as $\mathbf{W}=$ $\boldsymbol{\Phi} \boldsymbol{\Sigma}^{1 / 2} \boldsymbol{\Gamma}$, where $\boldsymbol{\Phi}$ is the eigenvector matrix of $\mathbf{R}_{T}, \boldsymbol{\Sigma}$ is a diagonal matrix called power loading matrix whose entries should be computed for optimality, and $\boldsymbol{\Gamma}$ is an arbitrary unitary matrix.

Proof. By taking the expectation in (13) and eigendecompositions $\mathbf{W} \mathbf{W}^{H}=\boldsymbol{\Psi} \boldsymbol{\Sigma} \boldsymbol{\Psi}^{H}$ and $\mathbf{R}_{T}=\boldsymbol{\Phi} \boldsymbol{\Delta} \boldsymbol{\Phi}^{H}$, we obtain

$$
C \leq C_{\mathrm{UB}}=\log _{2}\left(\operatorname{det}\left(\mathbf{I}_{M}+\frac{\kappa}{\sigma_{n}^{2}} \boldsymbol{\Psi} \boldsymbol{\Sigma} \boldsymbol{\Psi}^{H} \boldsymbol{\Phi} \boldsymbol{\Delta} \boldsymbol{\Phi}^{H}\right)\right),
$$

where $\kappa$ is a constant that can be calculated by taking the expectation of the components of $\mathbf{G}$. Our aim is to find $\mathbf{W}$ that maximizes (14) under the power constraint, that is,

$$
\max \log _{2}\left(\operatorname{det}\left(\mathbf{I}_{M}+\frac{\kappa}{\sigma_{n}^{2}} \boldsymbol{\Psi} \boldsymbol{\Sigma} \boldsymbol{\Psi}^{H} \boldsymbol{\Phi} \boldsymbol{\Delta} \boldsymbol{\Phi}^{H}\right)\right) \quad \text { s.t. } \operatorname{tr}(\boldsymbol{\Sigma}) \leq P \text {. }
$$

Note that $\operatorname{tr}\left\{\mathbf{W} \mathbf{W}^{H}\right\} \leq P$ will directly result in $\operatorname{tr}\{\boldsymbol{\Sigma}\} \leq P$. Using Hadamard's inequality [16], the above optimization can be achieved when the argument of the determinant is a diagonal matrix. To this end, we should have $\boldsymbol{\Psi}=\boldsymbol{\Phi}$. In other words, the singular matrix of the precoder matrix should be the same as the singular matrix of transmit correlation matrix. Therefore, the precoder structure can be written as

$$
\mathbf{W}=\Phi \Sigma^{1 / 2} \Gamma,
$$

where $\Gamma$ is an arbitrary unitary matrix that has no effect on the system performance, and therefore can be set to identity for simplicity and $\boldsymbol{\Sigma}$ (the eigenvalue matrix of $\mathbf{W}$ ) is the power loading matrix that should be optimized.

By substituting (16) into (13), the optimization problem can be rewritten as

$$
\max _{\Sigma} \log _{2}\left(\operatorname{det}\left(\mathbf{I}+\frac{\kappa}{\sigma_{n}^{2}} \boldsymbol{\Sigma} \boldsymbol{\Delta}\right)\right) \quad \text { s.t. } \operatorname{tr}(\boldsymbol{\Sigma}) \leq P,
$$

with the following solution for elements of $\Sigma$ :

$$
\sigma_{i}=\left[v-\frac{\sigma_{n}^{2}}{\kappa \delta_{i}}\right]^{+}, \quad i=0: M-1,
$$

where $[x]^{+}=\max [0, x]$ for a scalar $x, \sigma_{i}$ and $\delta_{i}$ are the diagonal entries of $\boldsymbol{\Sigma}$ and $\Delta$, respectively and $v$ is the constant determined by the power constraint. At the optimum point, the power inequality $\operatorname{tr}\{\boldsymbol{\Sigma}\} \leq P$ becomes equality.

In fact, the precoder changes the eigenvalues of the channel to optimize the ergodic capacity. The new eigenvalues of the product of the channel matrix and precoder matrix are $\sigma_{i} \delta_{i}, i=0 \cdots M-1$ (instead of $\delta_{i}$ ). Precoder tends to increase the larger eigenvalues compared to small eigenvalues and increase the eigenvalue spread of the product matrix HW. Therefore, $\delta_{i}>\delta_{j}$ results in $\sigma_{i}>\sigma_{j}$. This power allocation process is known as waterpouring in which the precoder pours more power to stronger eigenvalues (or eigenmodes) and allocates less to weaker ones.

\section{FREQUENCY-SELECTIVE FADING CHANNEL}

\subsection{System model}

We consider a transmission system with $M$ transmit and $N$ receive antennas in a frequency-selective fading channel. Because of the delay spread in the frequency-selective fading channel, the received signal is a function of the input signal at different time instants. The frequency-selective fading channel can be modeled as an $L$-tap FIR filter shown in Figure 1, and each tap denotes a resolvable channel path represented by an $N \times M$ matrix $\mathbf{H}_{l}, l=0, \ldots,(L-1)$. 


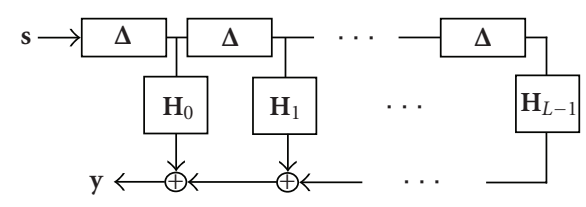

FIGURE 1: Frequency-selective MIMO channel model.

Consider a transmitted block of $K+L$ vectors of size $M \times$ 1 , organized as a long $(K+L) M \times 1$ vector, where $K$ is an arbitrary value, $\overline{\mathbf{s}}(k)=[\mathbf{s}(k(K+L)), \ldots, \mathbf{s}(k(K+L)+K+L-$ $1)]^{T}$. At the receiver, we eliminated the first $L$ vectors of size $N \times 1$ to remove the interblock interference (IBI), and stack $K$ remaining received vectors of size $M \times 1$ to form a long $K M \times 1$ vector, $\overline{\mathbf{y}}(k)=[\mathbf{y}(k(K+L)+L), \ldots, \mathbf{y}(k(K+L)+K+$ $L-1)]^{T}$,

$$
\overline{\mathbf{y}}(k)=\overline{\mathbf{H}} \cdot \overline{\mathbf{s}}(k)+\overline{\mathbf{n}}(k),
$$

where $\overline{\mathbf{n}}(k)=[\mathbf{n}(k(K+L)+L), \ldots, \mathbf{n}(k(K+L)+K+L-1)]^{T}$ is the long $K M \times 1$ vector of $K$ subsequent noise vectors of size $M \times 1$, and $\overline{\mathbf{H}}$ is the $N K \times M(K+L)$ block-Toeplitz channel matrix:

$$
\overline{\mathbf{H}}=\left[\begin{array}{ccccccc}
\mathbf{H}_{L-1} & \mathbf{H}_{L-2} & \cdots & \mathbf{H}_{0} & 0 & \cdots & 0 \\
0 & \mathbf{H}_{L-1} & \mathbf{H}_{L-2} & \cdots & \mathbf{H}_{0} & \cdots & 0 \\
\vdots & \vdots & \vdots & \vdots & \vdots & \vdots & \vdots \\
0 & \cdots & \mathbf{H}_{L-1} & \mathbf{H}_{L-2} & \cdots & \mathbf{H}_{0} & 0 \\
0 & \cdots & 0 & \mathbf{H}_{L-1} & \mathbf{H}_{L-2} & \cdots & \mathbf{H}_{0}
\end{array}\right]
$$

The $N \times M$ matrix $\mathbf{H}_{l}(k)$ represents the spatial response corresponding to the resolvable channel path $l, l=0,1, \ldots, L-1$, at the instant $k$. Its entry, $h_{n m}(k)$ is the complex-valued random gain from the $m$ th transmit to $n$th receive antennas over the effective path $l$ at the instant $k$, assumed to be unchanged during a frame transmission. Assuming that the receive correlation matrix is identity for all paths, the channel path matrix $\mathbf{H}_{l}(k)$ can be written as

$$
\mathbf{H}_{l}(k)=\mathbf{G}_{l}(k) \mathbf{R}_{T, l}^{1 / 2}, \quad l=0: L-1 .
$$

Note that the spatial correlation matrix is a function of transmit antennas (such as antenna spacing and antenna pattern) and channel physical characteristics (angular spread and power angular spread). The former parameter is the same for all paths while the latter is different from one path to another. This results in different channel path transmit correlation matrices $\mathbf{R}_{T, l}, l=0 \cdots L-1$. We assume that the power of the $l$ th path has been considered in the diagonal entries of its spatial correlation matrix $\mathbf{R}_{T, l}$. Due to the different delays between $L$ effective paths, (20) can provide a frequency-selective fading MIMO channel model, while each individual $\mathbf{H}_{l}(k)$ just represents a frequency-flat fading MIMO channel.
The block-Toeplitz channel matrix in (20) can be written as

$\overline{\mathbf{H}}=\left[\begin{array}{c}\mathbf{H} \\ \mathbf{H E} \\ \mathbf{H E}^{2} \\ \vdots \\ \mathbf{H E}^{K-1}\end{array}\right]=\left(\mathbf{I}_{K} \otimes \mathbf{H}\right) \cdot\left[\begin{array}{c}\mathbf{I}_{M(K+L)} \\ \mathbf{E} \\ \mathbf{E}^{2} \\ \vdots \\ \mathbf{E}^{K-1}\end{array}\right]=\left(\mathbf{I}_{K} \otimes \mathbf{H}\right) \cdot \overline{\mathbf{E}}$

where $\otimes$ stands for Kronecker product, the $N \times M(K+L)$ matrix $\mathbf{H}=\left[\mathbf{H}_{L-1}, \mathbf{H}_{L-2}, \ldots, \mathbf{H}_{0}, 0, \ldots, 0\right]$ is the first row of $\overline{\mathbf{H}}$ in (20), and $\mathbf{I}_{K}$ is the $K \times K$ identity matrix. The $M(K+$ $L) \times M(K+L)$ matrix $\mathbf{E}$ is a column switching matrix and has the following structure:

$$
\mathbf{E}=\left[\begin{array}{cc}
\mathbf{0}_{M(K+L-1) \times M} & \mathbf{I}_{M(K+L-1)} \\
\mathbf{I}_{M} & \mathbf{0}_{M \times M(K+L-1)}
\end{array}\right],
$$

where $\mathbf{0}_{M(K+L-1) \times M}$ and $\mathbf{0}_{M \times M(K+L-1)}$ are the $M(K+L-1) \times M$ and $M \times M(K+L-1)$ zero-matrices, respectively. We can verify the following properties of $\mathbf{E}^{i}, i=0,1, \ldots, K-1$.

(i) $\mathbf{E}^{i}$ can be obtained by applying column switching in an identity matrix, and $\mathbf{E}^{i}\left(\mathbf{E}^{i}\right)^{T}=\mathbf{I}$. Therefore, the eigenvalues of $\mathbf{I}$ and $\mathbf{E}^{i}$ have the same absolute values, and $\operatorname{det}\left(\mathbf{E}^{i}\right)= \pm 1$.

(ii) For an arbitrary $M(K+L) \times M(K+L)$ matrix $\mathbf{A}$ that can be eigendecomposed as $\mathbf{A}=\mathbf{U} \mathbf{\Lambda} \mathbf{U}^{H}$ and $\mathbf{A E}^{i}=$ $\mathbf{U}_{1} \Lambda_{1} \mathbf{U}_{1}^{H}$, it follows that $\Lambda_{1}=\Lambda$ and $\mathbf{U}_{1}=\mathbf{U E}^{i}$.

From the above properties, (21), and (22), the channel model can be written as

$$
\overline{\mathbf{H}}=\left(\mathbf{I}_{K} \otimes \mathbf{G R}_{T}^{1 / 2}\right) \overline{\mathbf{E}}=\left(\mathbf{I}_{K} \otimes \mathbf{G}\right)\left(\mathbf{I}_{K} \otimes \mathbf{R}_{T}^{1 / 2}\right) \overline{\mathbf{E}},
$$

where $\mathbf{G}=\left[\mathbf{G}_{L-1}, \mathbf{G}_{L-2}, \ldots, \mathbf{G}_{0}, 0, \ldots, 0\right]$ is an $N \times M(K+L)$ matrix whose elements $\mathbf{G}_{l}$ 's are $N \times M$ matrices with i.i.d. zero-mean complex Gaussian entries and 1/2 variance per dimension. The remaining entries are zero, that is, $\mathbf{0}_{N \times M}$ denotes an $N \times M$ zero matrix. Furthermore, $\mathbf{R}_{T}$ is the $M(K+L) \times M(K+L)$ transmit correlation matrix with the following structures:

$$
\mathbf{R}_{T}=\left[\begin{array}{ccccccc}
\mathbf{R}_{T, L-1} & & & & & \\
& \mathbf{R}_{T, L-2} & & & & 0 & \\
& & \ddots & & & & \\
& & & \mathbf{R}_{T, 0} & & \\
& & & & 0 & & \\
& 0 & & & & \ddots & \\
& & & & & & 0
\end{array}\right] \text {, }
$$

where $\mathbf{R}_{T, l}$ is the $M \times M$ transmit correlation matrix associated with the $l$ th channel path as defined in (21).

\subsection{Effect of correlation}

The following lemma sheds some light on the effect of transmit correlation matrices on the ergodic capacity of frequency-selective MIMO channel. 
Lemma 4. The upper bound on ergodic capacity of a frequency-selective channel is a function of a matrix representing the sum of the eigenvalue matrices of spatial correlation matrices of different paths, $\overline{\boldsymbol{\Lambda}}=\sum_{i=0}^{L-1}\left(\mathbf{E}^{i}\right)^{T} \operatorname{diag}\left(\boldsymbol{\Delta}_{l}\right) \mathbf{E}^{i}$.

Starting by the mutual information equation for frequencyselective channel [17-19], write

$$
I(\overline{\mathbf{s}} ; \overline{\mathbf{y}})=\frac{1}{P} \log \left[\operatorname{det}\left(\mathbf{I}_{M(K+L)}+\frac{P}{N K \sigma_{n}^{2}} \overline{\mathbf{H}}^{H} \overline{\mathbf{H}}\right)\right] .
$$

Subsequently, for sufficiently large $P$, the ergodic capacity of a frequency-selective fading channel is

$$
C=E\{I(\overline{\mathbf{s}} ; \overline{\mathbf{y}})\}=\frac{1}{P} E\left\{\log \left[\operatorname{det}\left(\mathbf{I}_{M(K+L)}+\frac{P}{N K \sigma_{n}^{2}} \overline{\mathbf{H}}^{H} \overline{\mathbf{H}}\right)\right]\right\} .
$$

Using the eigenvalue decomposition $\mathbf{R}_{T}=\operatorname{diag}\left(\boldsymbol{\Phi}_{l} \boldsymbol{\Delta}_{l} \boldsymbol{\Phi}_{l}^{H}\right), l=$ $0, \ldots,(L-1)$, and $(24)$ for $\overline{\mathbf{H}}$, one obtains

$$
\begin{aligned}
\overline{\mathbf{H}}^{H} \overline{\mathbf{H}} & =\overline{\mathbf{E}}^{T}\left(\mathbf{I}_{K} \otimes \mathbf{G}^{H} \operatorname{diag}\left(\boldsymbol{\Phi}_{l}\right) \operatorname{diag}\left(\boldsymbol{\Delta}_{l}\right) \operatorname{diag}\left(\boldsymbol{\Phi}_{l}^{H}\right) \mathbf{G}\right) \overline{\mathbf{E}} \\
& \sim \overline{\mathbf{E}}^{T}\left(\mathbf{I}_{K} \otimes \mathbf{G}^{H} \operatorname{diag}\left(\boldsymbol{\Delta}_{l}\right) \mathbf{G}\right) \overline{\mathbf{E}}
\end{aligned}
$$

since $\operatorname{diag}\left(\boldsymbol{\Phi}_{l}^{H}\right) \mathbf{G} \sim \mathbf{G}$ and $\mathbf{G}^{H} \operatorname{diag}\left(\boldsymbol{\Phi}_{l}\right) \sim \mathbf{G}^{H}$. The ergodic capacity of a frequency-selective fading channel in (27) can now be rewritten as

$$
\begin{aligned}
C \approx \frac{1}{P} E\{\log [\operatorname{det}( & \mathbf{I}_{M(K+L)} \\
& \left.\left.\left.+\frac{P}{N K \sigma_{n}^{2}} \overline{\mathbf{E}}^{T}\left(\mathbf{I}_{K} \otimes \mathbf{G}^{H} \operatorname{diag}\left(\Delta_{l}\right) \mathbf{G}\right) \overline{\mathbf{E}}\right)\right]\right\} .
\end{aligned}
$$

By using Jensen's inequality and taking the expectation, derive an upper bound on (29) as

$$
\begin{aligned}
C \leq & C_{\mathrm{UB}} \\
=\frac{1}{P} \log _{2} \operatorname{det} E\left\{\mathbf{I}_{M(K+L)}\right. & \left.\quad+\frac{P}{N K \sigma_{n}^{2}} \overline{\mathbf{E}}^{T}\left(\mathbf{I}_{K} \otimes \mathbf{G}^{H} \operatorname{diag}\left(\Delta_{l}\right) \mathbf{G}\right) \overline{\mathbf{E}}\right\} \\
& \quad \\
& \quad \frac{1}{P} \log _{2} \operatorname{det}\left(\mathbf{I}_{M(K+L)}+\frac{P}{K \sigma_{n}^{2}} \overline{\mathbf{E}}^{T}\left(\mathbf{I}_{K} \otimes \operatorname{diag}\left(\Delta_{l}\right)\right) \overline{\mathbf{E}}\right) .
\end{aligned}
$$

The right-hand side matrices in (30) can be multiplied, and hence it can be written as the sum of $K$ products:

$C \leq C_{\mathrm{UB}}=\frac{1}{P} \log _{2} \operatorname{det}\left(\mathbf{I}_{M(K+L)}+\frac{P}{K \sigma_{n}^{2}} \sum_{i=0}^{L-1}\left(\mathbf{E}^{i}\right)^{T} \operatorname{diag}\left(\boldsymbol{\Delta}_{l}\right) \mathbf{E}^{i}\right)$,

where $\mathbf{E}^{i}(i=0 \cdots L-1)$ denote the column-shifted versions of $\mathbf{E}$ defined in (23). Therefore, the upper bound on ergodic capacity of a frequency-selective channel is a function of the sum of eigenvalue matrices of spatial correlation matrices of different paths, that is, $\overline{\boldsymbol{\Lambda}}=\sum_{i=0}^{L-1}\left(\mathbf{E}^{i}\right)^{T} \operatorname{diag}\left(\boldsymbol{\Delta}_{l}\right) \mathbf{E}^{i}$.
Lemma 4 shows the importance of the eigenvalues of the spatial correlation matrices (of different paths in a frequencyselective fading channel) in the upper bound on ergodic capacity, and hence in ergodic capacity itself. The exact analysis of the effect of eigenvalue matrices on the ergodic capacity is however not easy. Nevertheless, generally, when the correlation matrices are such that matrix $\bar{\Lambda}$ is a scaled identity matrix, the most convenient case is of course when there is no spatial correlation for different paths, that is, when all the eigenvalues are one $\left(\Delta_{l}=(1 / M) \mathbf{I}_{M},(l=0 \cdots L-1)\right)$, yet we can also find other cases that correlation matrices are not identity but the ergodic capacity of the channel is maximized.

\subsection{Precoder design}

Our objective in this subsection is to find the optimal precoder matrix $\mathbf{W}$, to maximize the ergodic capacity in (27) for frequency-selective channel based on the partial channel knowledge of only the spatial correlation matrices $\mathbf{R}_{T, l}$ $(l=0 \cdots L-1)$ available at the transmitter. Recall that the precoding matrix at the transmitter is only needed to recompute over a long interval whenever the spatial correlation matrices are changed. This point makes this precoder suitable for the channels with fast fading.

Lemma 5 specifies the structure of the precoder in this case.

Lemma 5. The $M(K+L) \times M(K+L)$ linear precoding matrix $\mathbf{W}$ that maximizes the ergodic capacity of a frequencyselective fading channel of (24) is a block diagonal matrix $\mathbf{W}=\operatorname{diag}\left(\mathbf{W}_{i}\right)$, with $(K+L)$ optimal $M \times M$ matrices $\mathbf{W}_{i}=$ $\boldsymbol{\Phi}_{i} \Sigma_{i}^{1 / 2} \boldsymbol{\Gamma}_{i}$, where $\boldsymbol{\Gamma}_{i}$ 's are $M \times M$ arbitrary unitary matrices, $\boldsymbol{\Sigma}_{i}$ 's are diagonal matrices, and $\boldsymbol{\Phi}_{i}$ 's are the $M \times M$ unitary matrices resulting from eigendecomposition of transmit correlation matrices $\mathbf{R}_{T, l}$ 's, $l=0,1, \ldots,(L-1)$.

Proof. Based on (27), the ergodic capacity of the system using the precoder can be written as

$$
C=\frac{1}{P} E\left\{\log _{2} \operatorname{det}\left(\mathbf{I}_{M(K+L)}+\frac{P}{N K \sigma_{n}^{2}} \mathbf{W}^{H} \overline{\mathbf{H}}^{H} \overline{\mathbf{H}} \mathbf{W}\right)\right\} .
$$

Following the same steps as in the previous case, we can derive the upper bound on ergodic capacity as

$$
C \leq C_{\mathrm{UB}}=\frac{1}{P} \log _{2} \operatorname{det}\left(\mathbf{I}_{M(K+L)}+\frac{P}{K \sigma_{n}^{2}} \overline{\mathbf{R}}_{T} \boldsymbol{\Psi} \Sigma \Psi^{H}\right),
$$

where $\mathbf{R}_{T}$ is defined in (25), and $\overline{\mathbf{R}}_{T}=\sum_{l=1}^{K-1}\left(\mathbf{E}^{l}\right)^{T} \mathbf{R}_{T} \mathbf{E}^{l}$. Note that $\overline{\mathbf{R}}_{T}$ is also a block diagonal matrix.

Considering that $\overline{\mathbf{R}}_{T}=\operatorname{diag}\left(\overline{\boldsymbol{\Phi}}_{i} \overline{\boldsymbol{\Delta}}_{i} \overline{\boldsymbol{\Phi}}_{i}^{H}\right), i=0,1, \ldots,(K+$ $L-1)$, and using $\operatorname{det}(\mathbf{I}+\mathbf{A B})=\operatorname{det}(\mathbf{I}+\mathbf{B A})$, one can find $\boldsymbol{\Psi}=$ $\operatorname{diag}\left(\overline{\boldsymbol{\Phi}}_{i}\right), i=0,1, \ldots,(K+L-1)$. Therefore, the precoding matrix can be written as

$$
\begin{aligned}
\mathbf{W} & =\operatorname{diag}\left(\overline{\boldsymbol{\Phi}}_{i}\right) \boldsymbol{\Sigma}^{1 / 2} \boldsymbol{\Gamma} \\
& =\operatorname{diag}\left(\overline{\boldsymbol{\Phi}}_{i} \boldsymbol{\Sigma}_{i}^{1 / 2} \boldsymbol{\Gamma}_{i}\right), \quad i=0,1, \ldots,(K+L-1),
\end{aligned}
$$




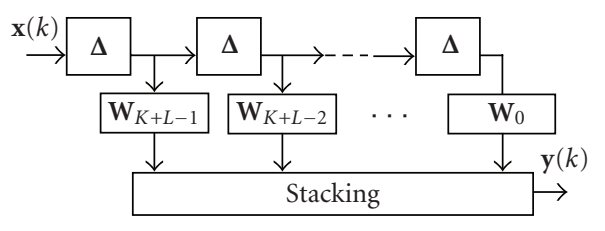

FIgURe 2: Precoder structure for a frequency-selective fading channel with $\mathrm{L}$ independent effective paths.

where $\Gamma_{i}$ is an arbitrary unitary matrix that can be set to identity for simplicity. Therefore, the transmit precoding matrix W is also a block diagonal matrix with $(K+L)$ optimal $M \times M$ matrices $\mathbf{W}_{i}=\overline{\boldsymbol{\Phi}}_{i} \boldsymbol{\Sigma}_{i}^{1 / 2} \boldsymbol{\Gamma}_{i}$, where $\overline{\boldsymbol{\Phi}}_{i}$ is one diagonal block of the eigenvector matrix of $\overline{\mathbf{R}}_{T}=\sum_{l=1}^{K-1}\left(\mathbf{E}^{l}\right)^{T} \mathbf{R}_{T} \mathbf{E}^{l}$.

Lemma 5 shows the structure of the optimal precoder matrix in this case. Applying this precoder matrix changes the eigenvalues of the correlation matrices of the channel from $\left(\mathbf{E}^{i}\right)^{T} \operatorname{diag}\left(\boldsymbol{\Delta}_{l}\right) \mathbf{E}^{i},(i=0 \cdots L-1)$ to $\boldsymbol{\Sigma}_{i}\left(\mathbf{E}^{i}\right)^{T} \operatorname{diag}\left(\boldsymbol{\Delta}_{l}\right) \mathbf{E}^{i}$, $(i=0 \cdots L-1)$. It remains to find the diagonal entries of the multiplier matrices $\boldsymbol{\Sigma}_{i}$ 's $(i=0 \cdots L-1)$ to modify the eigenvalues in order to achieve the maximum upper bound on ergodic capacity in (33), that is,

$$
\begin{array}{r}
\max _{\boldsymbol{\Sigma}} \log _{2} \operatorname{det}\left(\mathbf{I}_{M(K+L)}+\frac{P}{K \sigma_{n}^{2}} \operatorname{diag}\left(\overline{\boldsymbol{\Delta}}_{i}\right) \boldsymbol{\Sigma}\right) \\
\text { s.t. } \operatorname{tr}(\boldsymbol{\Sigma}): \text { constant. }
\end{array}
$$

Solving (35) results in the following relation:

$$
\sigma_{i}=\left[\nu-\left(\frac{P}{K \sigma_{n}^{2}} \bar{\delta}_{(i \bmod M)}\right)^{-1}\right]^{+}, \quad i=1,2, \ldots, M(L+K),
$$

where $\sigma_{i}$ 's (called power loading coefficients) and $\bar{\delta}_{i}$ 's are the diagonal entries of $\boldsymbol{\Sigma}$ and $\overline{\boldsymbol{\Delta}}_{i}$, respectively, and $v$ is the constant determined by the power constraint. The waterpouring equation in this case is a function of $\bar{\Delta}_{i}$ the eigenvalue matrices of $\overline{\mathbf{R}}_{T}=\operatorname{diag}\left(\overline{\boldsymbol{\Phi}}_{i} \overline{\boldsymbol{\Delta}}_{i} \overline{\boldsymbol{\Phi}}_{i}^{H}\right), i=0,1, \ldots,(K+L-1)$. In other words, these equations are not directly related to transmit correlation matrix $\mathbf{R}_{T}$ defined in (25).

In other words, the precoding matrix for a frequencyselective fading channel with $L$ independent effective paths is block diagonal. Therefore, the corresponding structure can be decoupled into $(K+L) M \times M$ precoders for frequency-flat fading channels as shown in Figure $2 . \Delta$ blocks in the precoder structure are just time delays. The construction of the $(K+L)$ precoders requires to solve the eigendecomposition of an $M(K+L) \times M(K+L)$ matrix $\overline{\mathbf{R}}_{T}$, or equivalently $L$ different transmit correlation matrices of size $M \times M$.

\section{NUMERICAL RESULTS}

At first, we investigate the effect of eigenvalues of spatial correlation matrix on ergodic capacity of a frequency-selective channel. We consider a system with two receive antennas $(N=2)$ and different number of transmit antennas and



FIGURE 3: Performance comparison in partially correlated channels.

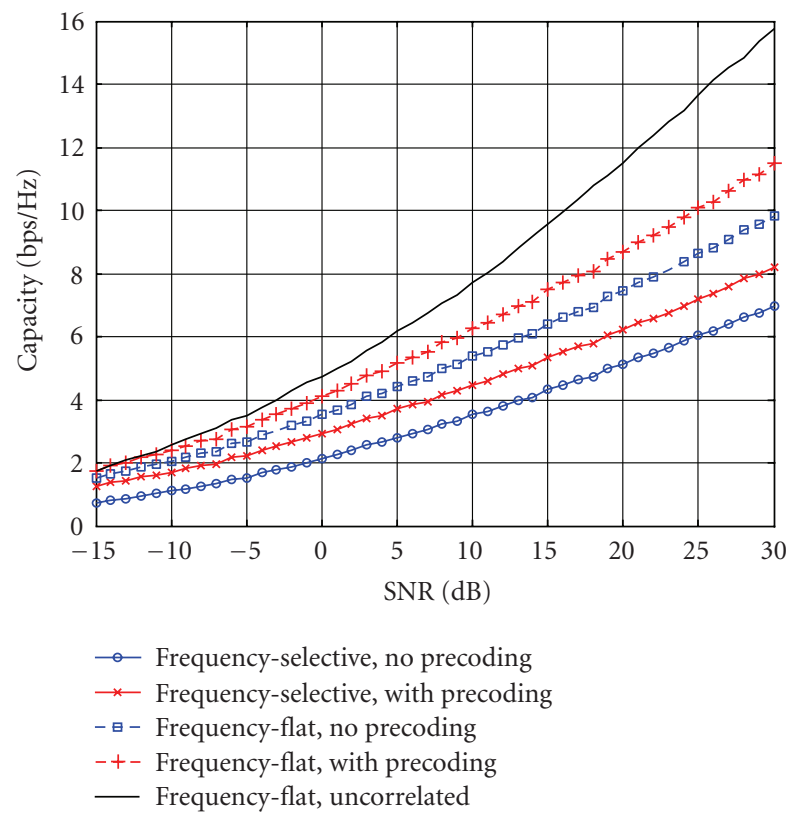

FIGURE 4: Performance comparison in fully correlated channels.

channel paths (i.e., $M=2,4$ and $L=2,4$ ). Figure 5 shows the ergodic capacity of the system for different eigenvalue spreads $\left(\lambda_{\max } / \lambda_{\min }\right): 1$ (no correlation), 2 (partial correlation), and $\infty$ (full correlation). The results clearly indicate that the capacity decreases with an increase in eigenvalue spread of the spatial correlation matrices.

Figure 6 compares the change in the eigenvalue spread of specific channels after applying linear precoding for different numbers of transmit antennas in frequency-flat and frequency-selective channels with two paths. Precoder increases the eigenvalue spread in the sense that it increases 




Figure 5: Ergodic capacity with different eigenvalue spreads and numbers of transmit antennas and channel paths.



FIGURE 6: Eigenvalue spread before and after applying precoding.

large eigenvalues (magnifies the strong eigenmodes) while it decreases small eigenvalues (weakens the weak eigenvalues) in order to improve ergodic capacity. The function of the precoders in changing the eigenvalues of spatial correlation matrices is also clear from (18) and (36).

Next, we investigate the precoder performance in two different cases of spatial correlation at the transmit and receive sides: (i) partial spatial correlation, that is, with eigenvalue spread close to unity, and full-rank correlation matrices,

(ii) full spatial correlation, that is, with very large eigenvalue spread, and rank-deficient spatial correlation matrices.

As an illustrative example, we consider a MIMO system with 2 transmit and 2 receive antennas $(M=N=2)$ in both frequency-flat and frequency-selective fading channels. The frequency-selective fading channel under consideration is represented by a 2-path model $(L=2)$. Furthermore, we assume that the channel paths are temporally uncorrelated.

Figures 3 and 4 illustrate the achievable capacity curves. For benchmark purpose, the capacity curve of an uncorrelated frequency-flat fading channel is also included. In both cases, precoders designed for frequency-flat and frequencyselective fading channels offer noticeable increases in the ergodic capacity of the system. In the case of partially correlated channel, the curves are closer to the uncorrelated frequency-flat fading case. On the other hand, the precoders perform better when the channels are highly spatially correlated.

\section{CONCLUDING REMARKS}

We investigated the importance of eigenvalues of spatial correlation matrices on the ergodic capacity of frequency-flat and -selective MIMO channels. We showed that the ergodic capacity depends greatly on the eigenvalue distribution of spatial correlation matrices. In other words, knowing the eigenstructure of correlation matrices at the transmitter is very important to enhance the capacity of the system. Based on this fact, we first investigated the effect of eigenvalues distribution of spatial and path correlation matrices on the capacity of frequency-flat and -selective channels. Next, we introduced a linear scheme known as linear precoding that can enhance the ergodic capacity of the channel by changing the eigenstructure of the channel by applying a linear transformation. We derived the structures of precoders using eigenvalue decomposition and linear algebra techniques in both cases and show their similarities from an algebraic point of view. Simulations showed the ability of this technique to change the eigenstructure of the channel, and hence to enhance the ergodic capacity considerably.

\section{REFERENCES}

[1] D. Chizhik, G. J. Foschini, M. J. Gans, and R. A. Valenzuela, "Keyholes, correlations, and capacities of multi-element transmit and receive antennas," IEEE Transactions on Wireless Communications, vol. 1, no. 2, pp. 361-368, 2002.

[2] D. Gesbert, H. Bölcskei, D. A. Gore, and A. J. Paulraj, "Outdoor MIMO wireless channels: models and performance prediction," IEEE Transactions on Communications, vol. 50, no. 12, pp. 1926-1934, 2002.

[3] J. Litva and T. K. Lo, Digital Beamforming in Wireless Communications, Artech House, Boston, Mass, USA, 1996. 
[4] A. O. Boukalov and S. G. Häggman, "System aspects of smartantenna technology in cellular wireless communications: an overview," IEEE Transactions on Microwave Theory and Techniques, vol. 48, no. 6, pp. 919-929, 2000.

[5] H. Sampath, P. Stoica, and A. J. Paulraj, "Generalized linear precoder and decoder design for MIMO channels using the weighted MMSE criterion," IEEE Transactions on Communications, vol. 49, no. 12, pp. 2198-2206, 2001.

[6] H. Sampath, Linear precoding and decoding for multiple input multiple output (MIMO) wireless channels, Ph.D. thesis, Stanford University, Stanford, Calif, USA, May 2001.

[7] A. Scaglione, P. Stoica, S. Barbarossa, G. B. Giannakis, and H. Sampath, "Optimal designs for space-time linear precoders and decoders," IEEE Transactions on Signal Processing, vol. 50, no. 5, pp. 1051-1064, 2002.

[8] H. Sampath and A. J. Paulraj, "Linear precoding for spacetime coded systems with known fading correlations," IEEE Communications Letters, vol. 6, no. 6, pp. 239-241, 2002.

[9] S. Zhou and G. B. Giannakis, "Optimal transmitter eigenbeamforming and space-time block coding based on channel correlations," IEEE Transactions on Information Theory, vol. 49, no. 7, pp. 1673-1690, 2003.

[10] H. R. Bahrami and T. Le-Ngoc, "Precoder design based on correlation matrices for MIMO systems," IEEE Transactions on Wireless Communications, vol. 5, no. 12, pp. 3579-3587, 2006.

[11] D. Gesbert, H. Bölcskei, D. A. Gore, and A. J. Paulraj, "Outdoor MIMO wireless channels: models and performance prediction," IEEE Transactions on Communications, vol. 50, no. 12, pp. 1926-1934, 2002.

[12] I. E. Telatar, "Capacity of multi-antenna Gaussian channel," Tech. Rep., Bell Labs, Murray Hills, NJ, USA, 1995.

[13] G. J. Foschini and M. J. Gans, "On limits of wireless communications in a fading environment when using multiple antennas," Wireless Personal Communications, vol. 6, no. 3, pp. 311-335, 1998.

[14] H. Bölcskei, D. Gesbert, and A. J. Paulraj, "On the capacity of OFDM-based spatial multiplexing systems," IEEE Transactions on Communications, vol. 50, no. 2, pp. 225-234, 2002.

[15] T. M. Cover and J. A. Thomas, Elements of Information Theory, John Wiley \& Sons, New York, NY, USA, 1991.

[16] R. A. Horn and C. R. Johnson, Matrix Analysis, Cambridge University Press, New York, NY, USA, 1985.

[17] G. G. Raleigh and J. M. Cioffi, "Spatio-temporal coding for wireless communication," IEEE Transactions on Communications, vol. 46, no. 3, pp. 357-366, 1998.

[18] V. Kafedziski, "Capacity of frequency selective fading channels with side information," in Proceedings of the 32nd Asilomar Conference on Signals, Systems and Computers, vol. 2, pp. 1758-1762, Pacific Grove, Calif, USA, November 1998.

[19] K. Liu, T. Kadous, and A. M. Sayeed, "Orthogonal timefrequency signaling over doubly dispersive channels," IEEE Transactions on Information Theory, vol. 50, no. 11, pp. 2583 2603, 2004 .

Hamid Reza Bahrami received his B.S. and M.S. degrees both in electrical engineering from Sharif University of Technology and University of Tehran in 2001 and 2003, respectively. He is currently a Ph.D. Candidate at McGill University. His research interest is in the area of wireless communications with emphasis on transmission techniques in MIMO systems.

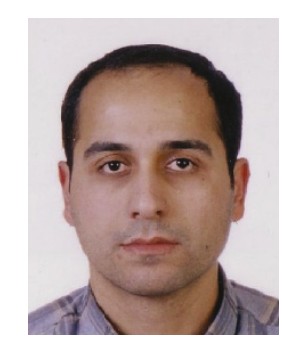

Tho Le-Ngoc obtained his B.Eng. degree (with distinction) in electrical engineering in 1976, his M.Eng. degree in microprocessor applications in 1978 from McGill University, Montreal, and his Ph.D. degree in digital communications in 1983 from the University of Ottawa, Canada. During 1977-1982, he was with Spar Aerospace Limited, involved in the development and

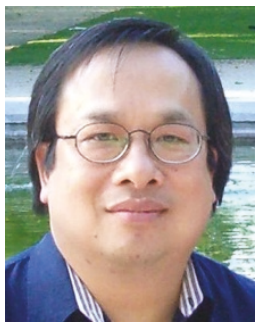
design of satellite communications systems.

During 1982-1985, he was an Engineering Manager of the Radio Group in the Department of Development Engineering of SRTelecom Inc., develop the new point-to-multipoint subscriber radio system SR500. During 1985-2000, he was a Professor at the Department of Electrical and Computer Engineering of Concordia University. Since 2000, he has been with the Department of Electrical and Computer Engineering of McGill University. His research interest is in the area of broadband digital communications with a special emphasis on modulation, coding, and multiple-access techniques. He is a Senior Member of the Ordre des Ingnieur du Quebec, a Fellow of the Institute of Electrical and Electronics Engineers (IEEE), a Fellow of the Engineering Institute of Canada (EIC), and a Fellow of the Canadian Academy of Engineering (CAE). He is the recipient of the 2004 Canadian Award in Telecommunications Research, and recipient of the IEEE Canada Fessenden Award 2005. 